Robert F. Warming and Richard M. Beam

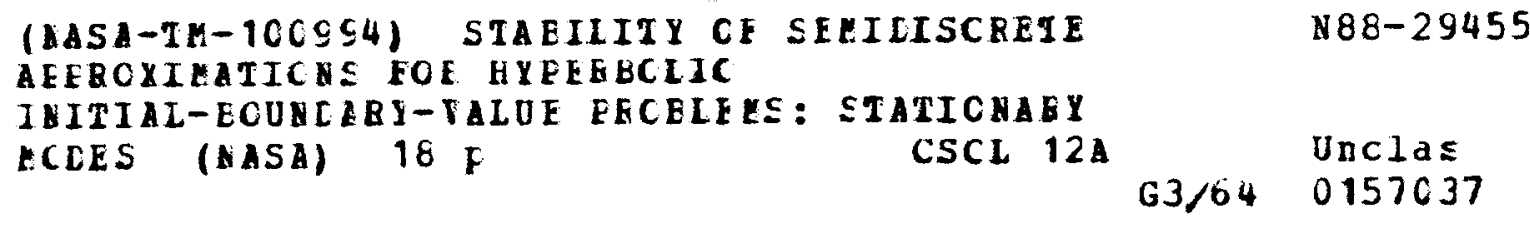

July 1988 


\section{Stability of Semidiscrete} Approximations for Hyperbolic Initial-Boundary-Value Problems: Stationary Modes

Robert F. Warming,

Richard M. Beam, Ames Research Center, Moffett Field, California

July 1988

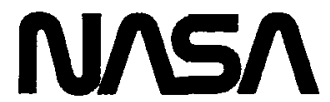

National Aeronautics and

Space Administration 


\title{
STABILITY OF SEMIDISCRETE APPROXIMATIONS FOR HYPERBOLIC INITIAL-BOUNDARY-VALUE PROBLEMS: STATIONARY MODES ${ }^{1}$
}

\author{
ROBERT F. WARMING AND RICHARD M. BEAM \\ NASA Ames Research Center \\ Moffett Field, CA 94035, USA
}

\begin{abstract}
Spatially discrete difference approximations of hyperbolic initial-boundaryvalue problems (IBVP's) require numerical boundary conditions in addition to the analytical boundary conditions specified for the differential equations. Improper treatment of a numerical boundary condition can cause instability of the discrete IBVP even though the approximation is stable for the pure initial-value or Cauchy problem. In the discrete IBVP stability literature there exists a small class of discrete approximations which are called borderline cases. For nondissipative approximations, borderline cases are unstable according to the theory of the Gustafsson, Kreiss, and Sundström (GKS) but they may be Lax-Richtmyer stable or unstable in the $L_{2}$ norm on a finite domain. We show that borderline approximations can be characterized by the presence of a stationary mode for the finite-domain problem. A stationary mode has the property that it does not decay with time and a nontrivial stationary mode leads to algebraic growth of the solution norm with mesh refinement. We give an analytical condition which makes it easy to detect a stationary mode, and we investigate several examples of numerical boundary conditions corresponding to borderline cases.
\end{abstract}

\section{INTRODUCTION}

In this paper we consider the stability of spatially discrete approximations to hyperbolic IBVP's. For simplicity we consider the stability of approximations to the IBVP for the model hyperbolic equation

$$
\frac{\partial u}{\partial t}=c \frac{\partial u}{\partial x}, \quad 0 \leq x \leq L, \quad t>0
$$

where $c$ is a real constant. One must specify initial data at $t=0$, and the IBVP is well-posed if an analytical boundary condition is prescribed at $x=L$

$$
u(L, t)=g(t) \text { for } \quad c>0 .
$$

A semidiscrete approximation of (1.1) is obtained by dividing the spatial interval into $J$ subintervals of length $\Delta x$ where $J \Delta x=L, x=x_{j}=j \Delta x$ and approximating

\footnotetext{
${ }^{1}$ Reprint for the Second International Conference on Hyperbolic Problems, Aachen, Fed. Rep. of Germany, March 14-18, 1988
} 
the spatial derivative $u_{x}$ by a difference quotient. As a prototype approximation, we replace $u_{x}$ by a second-order-accurate central-difference quotient to obtain a system of ordinary differential equations (ODE's)

$$
\frac{d u_{j}}{d t}=\frac{c}{2 \Delta x}\left(u_{j+1}-u_{j-1}\right), \quad j=1,2, \cdots, J-1
$$

where $u_{j}=u_{j}(t)$ denotes the approximation to $u(x, t)$. The right boundary $(x=L)$ is advanced by using the analytical boundary condition (1.2). For the stability analysis, we assume that the boundary condition is homogeneous, i.e., $g(t)=0$, and for the semidiscrete problem we write $u_{J}=0$.

A complication in completing the approximation is the fact that more boundary conditions are required for the semidiscrete approximation than are specified for the partial differential equation. If we apply (1.3) at the left boundary $(j=0)$, then the difference stencil protrudes one point to the left of the boundary. It is clear that that a numerical boundary condition is required. For example, at the left boundary $(j=0)$ we can change from a centered approximation to a one-sided approximation of $u_{x}$ :

$$
\frac{d u_{0}}{d t}=\frac{c}{\Delta x}\left[-\alpha u_{2}+(1+2 \alpha) u_{1}-(1+\alpha) u_{0}\right]
$$

where $\alpha$ is a parameter. Any procedure, e.g., (1.4), used to provide a numerical boundary condition is called a numerical boundary scheme (NBS). In the stability analysis of this paper it is convenient to express the NBS as a space extrapolation formula. The NBS (1.4) is equivalent to

$$
q(E) u_{-1}=0, \quad \text { where } \quad q(E)=(E-1)^{2}(2 \alpha E-1)
$$

and the shift operator $E$ is defined by

$$
E u_{j}=u_{j+1}
$$

The system of ODEs (1.3) together with the analytical boundary condition $u_{J}=0$ and the NBS (1.4) can be written in vector-matrix form as

$$
\frac{d \mathbf{u}(t)}{d t}=A \mathbf{u}(t)
$$

where $\mathbf{u}$ is a $J$-component vector and $A$ is a $J \times J$ matrix. The essential element in the stability of the semidiscrete approximation (1.7) is the behavior of the solution at a fixed time as the spatial mesh is refined. Consequently, one must consider an infinite sequence of ODE systems of dimension $J$ where $J \rightarrow \infty$ as $\Delta x \rightarrow 0$.

For the semidiscrete approximation (1.3) with periodic boundary conditions, the matrix $A=A_{p}$ is a skew-symmetric circulant matrix. Consequently the eigenvalues of $A_{p}$ are pure imaginary and the semidiscrete approximation is said to be nondissipative. The analysis of stationary modes for dissipative approximations will be given in a subsequent paper. 
Stability of a semidiscrete approximation with homogeneous boundary data means that there exists an estimate of the solution in terms of the initial data. For example, the semidiscrete approximation represented by the sequence of ODE's (1.7) is LaxRichtmyer stable if there exists a constant $K>0$ such that for any initial condition $\mathbf{u}(0)$

$$
\|\mathbf{u}(t)\| \leq K\|\mathbf{u}(0)\|
$$

for all $J>0, J \Delta x=L$ and for all $t, 0 \leq t \leq T$ with $T$ fixed. In this paper the symbol $\|\cdot\|$ denotes the discrete $L_{2}$ norm defined by

$$
\|\mathbf{u}(t)\|_{2}=\left(\sum_{j=0}^{J-1} u_{j}^{2} \Delta x\right)^{1 / 2} .
$$

Two methods for carrying out a stability analysis are the energy method and the normal mode analysis. The normal mode analysis is an eigenvalue analysis. If we look for a solution of (1.7) of the form $\mathbf{u}(t)=e^{s t} \phi$, then we obtain $A \phi=s \phi$. But this is just the eigenvalue-eigenvector problem for the matrix $A$ where $\phi$ is the eigenvector and $s$ is the eigenvalue. The practical problem of implementing stability tests on the eigenvalues is that the normal mode analysis for a discrete hyperbolic IBVP on a finite domain is, in general, analytically intractable.

The intractability can easily be demonstrated by the normal mode analysis of the ODE system (1.7). The components $\phi_{j}$ of the eigenvector $\phi$ and the normalized eigenvalue $\hat{s}=(\Delta x / c) s$ are given by

$$
\phi_{j}=a\left[\kappa^{j}-\left(-\kappa^{2}\right)^{J}(-1 / \kappa)^{j}\right], \quad 2 \hat{s}=\kappa-\frac{1}{\kappa}
$$

where $a$ is an arbitrary nonzero constant and $\kappa$ is a root of the characteristic equation

$$
q(\kappa)-\left(-\kappa^{2}\right)^{J+1} q(-1 / \kappa)=0 .
$$

The polynomial $q(\kappa)$ depends solely on the NBS, i.e., $q(\kappa)$ is the polynomial associated with the NBS written as an extrapolation formula. For example, the polynomial $q(\kappa)$ for the NBS (1.5) is

$$
q(\kappa)=(\kappa-1)^{2}(2 \alpha \kappa-1) .
$$

Since $J \Delta x=L$, the degree of (1.10c) increases as the spatial mesh increment $\Delta x$ decreases. In general, one cannot solve for the roots of (1.10c) which accounts for the analytic intractability of the normal mode analysis on a finite domain.

Although the Lax-Richtmyer condition (1.8) is a conventional stability definition, there is no known algebraic test to check the stability condition for discrete hyperbolic IBVP's on a finite domain. A more complicated stability definition is used in the theory developed by Gustafsson, Kreiss, and Sundström (GKS) [1]. Strikwerda [4] has extended the GKS theory to semidiscrete approximations. The advantage of the GKS theory accrues from the fact that a finite-domain problem with two boundaries is divided into a Cauchy problem and two quarter-plane problems each of which can 
be analyzed separately by a normal mode analysis. The analogues of (1.10) for the right-quarter plane problem are

$$
\phi_{j}=a \kappa^{j}, \quad 2 \hat{s}=\kappa-\frac{1}{\kappa}
$$

where $\kappa$ is a root of

$$
q(\kappa)=0 .
$$

This is the same polynomial $q(\kappa)$ that appears in (1.10c). The roots of $(1.12 \mathrm{c})$ are easily found since $q(\kappa)$ is of low degree. Algebraic tests based on the roots of $q(\kappa)$ and the corresponding eigenvalues $\hat{s}$ provide necessary and sufficient conditions for GKS stability.

The connection between the normal mode analysis for the finite-domain problem and the normal mode analysis for the quarter-plane problem is rather obscure. In a recent paper [6], we derived asymptotic estimates of the eigenvalues for the finitedomain problem. These estimates were used to relate the normal mode analysis of the finite-domain problem and the GKS quarter-plane analysis. In order to derive the asymptotic estimates for the roots of $(1.10 \mathrm{c})$, we assumed that particular roots can be identified for each $J$ and we write $\kappa=\kappa(J)$. Furthermore, there is no loss of generality in assuming that $|\kappa(J)| \leq 1$. We showed that the roots of the characteristic equation (1.10c) can be divided into three distinct classes according to the asymptotic behavior of $|\kappa(J)|^{J}$ in the limit $J \rightarrow \infty$. For $|\kappa(J)|<1$ there are only two possibilites:

$$
(I): \quad \lim _{J \rightarrow \infty}|\kappa(J)|^{J}=0, \quad(I I): \quad \lim _{J \rightarrow \infty}|\kappa(J)|^{J}=\text { constant }>0 .
$$

For roots in class $(I)$, it is clear that $(1.10 \mathrm{c})$ reduces to the quarter-plane equation (1.12c) as $J \rightarrow \infty$. Consequently, a root in class $(I)$ becomes a root of the quarterplane polynomial $(1.12 \mathrm{c})$ in the limit $J \rightarrow \infty$. The eigenvalues corresponding to the $\kappa$ 's of class $(I I)$ are benign in the stability analysis in the sense that they satisfy the inequality

$$
\Re(\hat{s}) \leq \frac{\text { const }}{\mathrm{J}}, \quad J \rightarrow \infty
$$

which is a necessary condition for stability (see [6]).

\section{STATIONARY MODES}

In addition to $(I)$ and $(I I)$, there is a third class of roots

$$
(I I I): \quad|\kappa(J)|=1
$$

If $\kappa$ is in class $(I I I)$ and is independent of $J$, then $\kappa$ remains fixed on the unit circle for all $J$. For this to happen it is obvious from (1.10c) that the polynomials $q(\kappa)$ and $q(-1 / \kappa)$ must have a common factor. This common factor leads to identical roots for both the quarter plane polynomial $q(\kappa)$ and the finite-domain characteristic equation $(1.10 \mathrm{c})$. These roots are fixed (independent of $J$ ) on the unit circle and from (1.10b) 
one obtains $\Re(\hat{s})=0$ with $\Im(\hat{s})=$ fixed. Consequently, there is a stationary mode, i.e., a mode with $\kappa$ and $\hat{s}$ independent of $J$.

If there is a stationary mode for the finite-domain problem, the GKS perturbation test will always indicate the presence of a GKS generalized eigenvalue and consequently the semidiscrete approximation is GKS unstable. The existence of a GKS generalized eigenvalue is easily proved. Since $|\kappa|=1$ and both $\kappa$ and $-1 / \kappa$ are roots of $q(\kappa)=0$, we need two perturbation tests, i.e.,

$$
\kappa_{a}^{*}=(1-\epsilon) e^{i \theta}, \quad \kappa_{b}^{*}=-(1-\epsilon) e^{-i \theta}
$$

where $\kappa_{a}^{*}$ and $\kappa_{b}^{*}$ denote the perturbations of $\kappa$ and $-1 / \kappa$ inside the unit circle. But from $(1.10 b)$ it follows that

$$
\Re\left[\hat{s}\left(\kappa_{a}^{*}\right)\right]=-\Re\left[\hat{s}\left(\kappa_{b}^{*}\right)\right] \neq 0
$$

and consequently there is a generalized eigenvalue $\Re\left(\hat{s}^{*}\right)>0$ corresponding to either $\kappa$ or $-1 / \kappa$.

The importance of a stationary mode is the following. Gustafsson et al. $[2]$ have proved that if the Cauchy stability requirement of the GKS theory is replaced by a more stringent energy estimate, then GKS stability implies Lax-Richtmyer stability in the $L_{2}$ norm. There is a small number of known examples showing that Lax-Richtmyer stability in the $L_{2}$ norm does not imply GKS stability. These examples all involve what are called borderline cases. We show that borderline cases have a stationary mode for the finite-domain problem. The GKS quarter plane analysis cannot detect whether or not a particular mode is stationary for the finite-domain problem. However, from our analysis, stationary modes are easy to detect since they occur if and only if $q(\kappa)$ and $q(-1 / \kappa)$ have a common factor.

From the point of view of an eigenvalue analysis, a semidiscrete approximation with a stationary mode must be treated separately since any instability derives not from an eigenvalue with a positive real part but from the algebraic growth (as $J \rightarrow \infty$ ) of the norm of the solution.

\section{EXAMPLES OF STATIONARY MODES}

In this section we examine several examples of stationary modes arising from various NBSs for semidiscrete approximations. We analyze the first example in detail but give only a summary of the stationary mode analysis for the subsequent examples. All of the examples are GKS unstable. Examples 1 and 2 are Lax-Richtmyer unstable but examples 3 and 4 are Lax-Richtmyer stable. In each of the examples we follow the convention of having the boundary condition of interest on the left boundary, i.e., the GKS analysis is done for a right quarter-plane problem.

\subsection{EXAMPLE 1}

Our first example of a semidiscrete approximation with a stationary mode is NBS (1.4) or equivalently (1.5) for $\alpha=-1 / 2$. The polynomial (1.11) becomes

$$
q(\kappa)=-\left(\kappa^{2}-1\right)(\kappa-1), \quad q(-1 / \kappa)=-\left(\kappa^{2}-1\right)(\kappa+1) \kappa^{3} .
$$


The polynomials $q(\kappa)$ and $q(-1 / \kappa)$ have the common factor $\left(\kappa^{2}-1\right)$, and consequently, there is a stationary mode. The characteristic equation (1.10c) has the roots

$$
\kappa= \pm 1
$$

independent of $J$ and, from (1.10b), $\hat{s}=0$. According to a GKS stability analysis, $\alpha=$ $-1 / 2$ is the borderline case between stability $(\alpha>-1 / 2)$ and instability $(\alpha<-1 / 2)$. For $\alpha=-1 / 2$ there is a stationary mode, and consequently, this borderline case is GKS unstable.

The NBS (1.4) with $\alpha=-1 / 2$ is

$$
\frac{d u_{0}}{d t}=\frac{c}{2 \Delta x}\left(u_{2}-u_{0}\right) .
$$

As an aid to interpreting the solution of a semidiscrete approximation with (3.3) as the NBS, it is useful to consider zeroth-order extrapolation

$$
u_{0}(t)=u_{1}(t)
$$

as an NBS. Differentiation of (3.4) with respect to time yields

$$
\frac{d u_{0}}{d t}=\frac{d u_{1}}{d t} .
$$

By replacing $d u_{1} / d t$ in the above equation by the interior approximation (1.3) evaluated at $j=1$, one obtains the NBS (3.3). If we integrate (3.5) from 0 to $t$ then

$$
u_{0}(t)-u_{1}(t)=u_{0}(0)-u_{1}(0)=\text { constant } .
$$

Hence, the NBS (3.3) is equivalent to zeroth-order space extrapolation if the initial value is reset at the outflow boundary so that

$$
u_{0}(0)-u_{1}(0)=0 .
$$

A semidiscrete approximation with zeroth-order extrapolation (3.4) as the NBS is both GKS stable and Lax-Richtmyer stable. However, differentiation of the NBS (3.4) yields an approximation which is both GKS unstable and Lax-Richmyer unstable. Next, we show that the Lax-Richtmyer instability is due to the presence of a stationary mode.

The stationary eigenvector corresponding to the stationary roots (3.2) is found by substitution of either $\kappa=1$ or -1 into (1.10a) to obtain

$$
\phi_{j}=a\left[1-(-1)^{j+J}\right], \quad j=0,1,, \cdots, J-1
$$

where $a$ is an arbitrary nonzero constant. The $L_{2}$ norm of the eigenvector (3.8) is

$$
\|\phi\|_{2}=\left(\sum_{j=0}^{J-1} \phi_{j}^{2} \Delta x\right)^{1 / 2}= \begin{cases}|a| \sqrt{2 L}, & J \text { even } \\ |a| \sqrt{2 L(J+1) / J}, & J \text { odd }\end{cases}
$$


where we have used $J \Delta x=L$.

The matrix $A$ corresponding to the ODE system (1.3) with (3.3) as the NBS and $u_{J}=0$ as the analytical boundary condition is $A=(c / \Delta x) \hat{A}$ where

$$
\widehat{A}=\frac{1}{2}\left[\begin{array}{rrrrrrrr}
-1 & 0 & 1 & & & & \\
-1 & 0 & 1 & & & O & \\
& \cdot & \cdot & . & & & \\
& & \cdot & . & . & & \\
& & & \cdot & . & . & \\
& 0 & & & -1 & 0 & 1 \\
& & & & & -1 & 0
\end{array}\right]
$$

Since the first two rows are equal, it is obvious that the matrix $\hat{A}$ has an eigenvalue $\hat{s}=$ 0 . The eigenvector (3.8) is the right eigenvector $\phi$ of the matrix (3.10) corresponding to $\hat{s}=0$. The left eigenvector $\boldsymbol{\xi}$ corresponding to $\hat{s}=0$ is easily found by inspection of the matrix (3.10) to be

$$
\left.\xi^{T}=\mid-1,1,0, \cdots, 0\right] .
$$

If we choose $a=1 / 2$ in (3.8), then the inner product of $\xi$ and $\phi$ is

$$
\xi^{T} \phi= \pm 1
$$

where the plus sign is used if $J$ is even and the minus sign if $J$ is odd.

The matrix (3.10) has a complete set of eigenvectors and consequently the general solution of the ODE system (1.7) can be written as

$$
\mathbf{u}(t)=\sum_{\ell=0}^{J-1} \beta_{\ell} e^{\varepsilon^{\prime} t} \phi(\ell)
$$

where $\phi(\ell)$ denotes the eigenvector corresponding to the eigenvalue $s_{\ell}$. We denote the stationary eigenvector (3.8) by $\phi(0)$ and rewrite (3.13) as

$$
\mathbf{u}(t)=\beta_{0} \phi(0)+\sum_{\ell=1}^{J-1} \beta_{\ell} e^{z_{\ell} t} \phi(\ell) .
$$

The sum on the right hand side of (3.14) consists of the eigenvalues and eigenvectors for zeroth-order space extrapolation (3.4) as the NBS.

For given initial data, the coefficient $\beta_{0}$ associated with the stationary eigenvector $\phi(0)$ is determined by taking the inner product of the left eigenvector $\xi$ given by (3.11) with $\mathbf{u}(0)$ given by $(3.14)$ :

$$
\boldsymbol{\xi}^{T} \mathbf{u}(0)=\left[-u_{0}(0)+u_{1}(0)\right]= \pm \beta_{0}
$$

where the plus sign corresponds to $J$ even and the minus sign to $J$ odd. In obtaining (3.15) we have made use of the fact that the right and left eigenvectors of a matrix are 
orthogonal. Formula (3.15) for the coefficient $\beta_{0}$ is consistent with our earlier assertion that the NBS (3.3) is equivalent to zeroth-order space extrapolation if the initial value is reset at the outfow boundary so that $u_{0}(0)-u_{1}(0)=0$.

One can show analytically that $\Re\left(s_{\ell}\right)<0$ for all the modes except the stationary mode, and consequently, the asymptotic solution $(t \rightarrow \infty)$ is from (3.14)

$$
\mathbf{u}(t) \approx \beta_{0} \phi(0), \quad t \rightarrow \infty .
$$

The $L_{2}$ norm of $(3.16)$ for $J$ even is

$$
\|\mathbf{u}(t)\|_{2} \approx\left|\beta_{0}\right|\|\phi(0)\|_{2} \approx\left|\beta_{0}\right| \sqrt{\frac{L}{2}}=\left|u_{0}(0)-u_{1}(0)\right| \sqrt{\frac{L}{2}}, \quad t \rightarrow \infty
$$

where we have used (3.9) with $a=1 / 2$ and (3.15).

The Lax-Richtmyer instability resulting from the NBS (3.3) arises from the presence of the stationary mode. We illustrate this on the finite domain $0 \leq x \leq L$ by choosing the initial data

$$
u_{j}(0)=\left\{\begin{array}{lll}
1 & \text { for } & j=0 \\
0 & \text { for } & j>0
\end{array}\right.
$$

where $J \Delta x=L$. The $L_{2}$ norm of this initial data is

$$
\|\mathbf{u}(0)\|_{2}=\Delta x^{1 / 2}=\sqrt{\frac{L}{J}} .
$$

For the initial data (3.18) with $J$ even, the $L_{2}$ norm of the asymptotic solution (3.17) is

$$
\|\mathbf{u}(t)\|_{2} \approx \sqrt{\frac{L}{2}}, \quad t \rightarrow \infty
$$

From (3.20) and (3.19) it follows

$$
\|\mathbf{u}(t)\|_{2} \approx \frac{\sqrt{J}}{\sqrt{2}}\|\mathbf{u}(0)\|_{2}, \quad t \rightarrow \infty .
$$

Consequently, the $L_{2}$ norm of the solution is not uniformly bounded on $0 \leq t \leq T$ (with $T$ large) for the initial data (3.18) and the semidiscrete approximation is Lax-Richtmyer unstable.

If the initial data are smooth, then the semidiscrete approximation converges although the global order of accuracy drops to first order. If we assume that the initial data are smooth, then

$$
u_{0}(0)-u_{1}(0)=f(0)-f(\Delta x)=-\Delta x \frac{\partial f(0)}{\partial x}+O\left(\Delta x^{2}\right) .
$$

In this case (3.17) becomes

$$
\|\mathbf{u}(t)\|_{2} \approx\left|\frac{\partial f(0)}{\partial x}\right| \sqrt{\frac{L}{2}} \Delta x=O(\Delta x), \quad t \rightarrow \infty .
$$




\section{2a EXAMPLE 2a}

In this section the model hyperbolic equation is

$$
\frac{\partial u}{\partial t}=-c \frac{\partial u}{\partial x}, \quad c>0, \quad 0 \leq x \leq L, \quad t>0,
$$

i.e., the analytical $\mathrm{BC}$ is specified at the left boundary, $x=0$. The spatial derivative $u_{x}$ is approximated by a centered approximation and the PDE (3.24) is replaced by the system of ODEs

$$
\frac{d u_{j}}{d t}=\frac{c}{2 \Delta x}\left(u_{j-1}-u_{j+1}\right), \quad j=1,2, \cdots, J-1 .
$$

The homogeneous analytical boundary condition is

$$
u_{0}=0 .
$$

The NBS is

$$
\frac{d u_{J}}{d t}=\frac{c}{\Delta x}\left(u_{J-1}-u_{J}\right) .
$$

The semidiscrete approximation (3.25) with boundary conditions (3.26) and (3.27) is both Lax-Richtmyer and GKS stable. In example 1 we showed that differentiation with respect to time of a stable NBS, i.e., zeroth-order extrapolation, resulted in an unstable semidiscrete approximation. In this section we show that differentiation of the analytical boundary condition (3.26) with respect to time, i.e.,

$$
\frac{d u_{0}}{d t}=0
$$

leads to an unstable approximation. The source of the instability is the introduction of a stationary mode. It should be noted that this example differs from the other examples in this paper since the stationary mode is introduced by an improper modification of the analytical boundary condition, i.e., replacement of (3.26) by (3.28), rather than an improper choice of the NBS.

It is easy to show by evaluating (3.25) at $j=0$, that (3.28) is equivalent to the extrapolation formula

$$
h(E) u_{-1}=0, \quad \text { where } h(E)=1-E^{2} .
$$

Since $h(\kappa)=1-\kappa^{2}$ it follows directly that

$$
h(-1 / \kappa)=-h(\kappa) / \kappa^{2}
$$

as one can easily verify. Therefore, $h(\kappa)$ and $h(-1 / \kappa)$ have a common factor, namely $h(\kappa)$ itself, and consequently there is a stationary mode. The roots of $h(\kappa)=0$ are $\kappa=$ \pm 1 . The stationary mode has the eigenvalue $\hat{s}=0$ and the corresponding eigenvector is

$$
\phi_{j}=a, \quad j=0,1,2, \cdots, J
$$


i.e., a vector with constant elements.

Since all the modes except the stationary mode are damped for large time $t$, the asymptotic solution is

$$
\mathbf{u}(t) \approx \beta_{0} \phi(0) \quad t \rightarrow \infty
$$

where $\beta_{0}=u_{0}(0)$ and the elements of $\phi(0)$ are given by (3.31). For the initial data (3.18) the asymptotic solution is

$$
\|\mathbf{u}(t)\|_{2} \approx \sqrt{L}=\sqrt{J}\|\mathbf{u}(0)\|_{2} \quad t \rightarrow \infty
$$

Consequently, the norm of the solution is not uniformly bounded for $0 \leq t \leq T$ (with $T$ large) for the initial data (3.18) and the semidiscrete approximation is Lax-Richtmyer unstable.

\section{2b EXAMPLE 2b}

We return to the model equation (1.1) with the NBS on the left boundary, $x=0$. If we choose the overspecified Dirichlet condition

$$
u_{0}=\mathbf{0}
$$

as the NBS, the resulting semidiscrete approximation is both Lax-Richtmyer and GKS stable. However, if we differentiate (3.34) with respect to time

$$
\frac{d u_{0}}{d t}=0
$$

the resulting approximation is both Lax-Richtmyer unstable and GKS unstable.

The NBS (3.35) is equivalent to the extrapolation formula

$$
q(E) u_{-1}=0, \text { where } q(E)=1-E^{2} .
$$

The polynomial $q(\kappa)=1-\kappa^{2}$, and therefore,

$$
q(-1 / \kappa)=-q(\kappa) / \kappa^{2} .
$$

Consequently, there is a stationary mode. For this example and the following examples, 3 and 4 , all of the roots of the characteristic equation are in class $(I I I)$, i.e., $|\kappa(J)|=1$ and all the eigenvalues are pure imaginary. Consequently, the normal modes for the finite-domain problem can be found analytically. For $J$ even the stationary mode has a repeated eigenvalue $\hat{s}=0$. For sufficiently large time the asymptotic solution is

$$
u_{j}(t) \approx\left\{\begin{array}{cl}
\frac{c t}{L}, & j=\text { odd } \\
0, & j=\text { even }
\end{array}, \quad t \rightarrow \infty\right.
$$

For the initial data (3.18), the norm of the asymptotic solution is

$$
\|\mathbf{u}(t)\|_{2} \approx \frac{\sqrt{J}}{\sqrt{2}} \frac{|c| t}{L}\|\mathbf{u}(0)\|_{2}, \quad t \rightarrow \infty .
$$


The Lax-Richtmyer instability arises from the factor $\sqrt{J}$ and not from the linear growth in $t$.

\subsection{EXAMPLE 3}

Consider the inconsistent NBS

$$
u_{-1}=-u_{1}
$$

which can be written as

$$
q(E) u_{-1}=0, \text { where } \quad q(E)=1+E^{2} .
$$

This NBS was used by Trefethen [5] for the leap frog scheme as an example of a fully discrete approximation to (1.1) that is Lax-Richtmyer stable and GKS unstable. As outlined below the same stability results are obtained when the NBS (3.40) is used for the semidiscrete approximation (1.3).

Since $q(\kappa)=1+\kappa^{2}$, one has

$$
q(-1 / \kappa)=q(\kappa) / \kappa^{2}
$$

and consequently, there is a stationary mode. From (1.10c) one obtains

$$
q(\kappa)\left[1+\left(-\kappa^{2}\right)^{J}\right]=0 .
$$

The roots of the equation (3.43) are determined from

$$
q(\kappa)=1+\kappa^{2}=0, \quad \text { and } \quad 1+\left(-\kappa^{2}\right)^{J}=0 .
$$

For the stationary mode the roots of $q(\kappa)=0$ are $\kappa= \pm i$ and the corresponding eigenvalues are $\hat{s}= \pm i$. However from (1.10b) the roots $\kappa= \pm i$ both lead to trivial stationary eigenvectors for the finite-domain problem.

For this example the eigensolutions of the finite-domain problem can be found analytically. The remaining roots of the characteristic equation can be determined from (3.44b) by using the roots of unity formula. The corresponding eigenvalues are pure imaginary. In particular, for $J$ odd the eigenvalues are

$$
\hat{s}_{\ell}=i \sin \left(\frac{2 \ell \pi}{J}\right), \quad \ell=1,2, \cdots, J \quad(J \text { odd }) .
$$

Here we have the rather amazing result that for $J$ odd, the eigenvalues are analytically identical to those of the circulant matrix associated with the spatially periodic problem. The eigenvectors are, of course, different.

One can show by the energy method that the semidiscrete approximation is LaxRichtmyer stable. In particular

$$
\|\mathbf{u}(t)\|_{2} \leq \sqrt{2}\|\mathbf{u}(0)\|_{2} .
$$


On the other hand, the approximation is GKS unstable because there is a stationary mode.

We briefly outline the GKS perturbation analysis. For $\kappa=i$, the eigenvalue is $\hat{s}=i$ where obviously $\Re(\hat{s})=0$ and hence we must check to see if there is a GKS generalized eigensolution. Let $\kappa^{*}$ denote a perturbation of $\kappa=i$ which is inside the unit circle, i.e.,

$$
\kappa=e^{i \theta}(1-\epsilon), \quad \theta=\frac{\pi}{2}+\epsilon, \quad \epsilon>0 .
$$

By inserting (3.47) into (1.10b), we find

$$
\Re\left(\hat{s}^{*}\right)=\epsilon^{2}+O\left(\epsilon^{3}\right) .
$$

Since the perturbation of $\Re(\hat{\mathcal{s}})$ is positive there is a GKS generalized eigenvalue and semidiscrete approximation is GKS unstable. The fact that an $\epsilon$ perturbation of $\kappa$ yields an $\epsilon^{2}$ perturbation in $\mathfrak{R}(\hat{s})$ indicates a weaker type of GKS instability than for a conventional GKS generalized eigenvalue where the perturbation in $\kappa$ and $\Re(\hat{s})$ are of the same order.

\subsection{EXAMPLE 4}

Our last example is due to Gustafsson[3]. The wave equation, $u_{t t}=u_{x x}$, written as a first-order system is

$$
\frac{\partial \mathrm{w}}{\partial t}=B \frac{\partial \mathrm{w}}{\partial x}, \quad \mathbf{w}=\left[\begin{array}{l}
u \\
v
\end{array}\right], \quad B=\left[\begin{array}{ll}
0 & 1 \\
1 & 0
\end{array}\right]
$$

The initial condition is $\mathbf{w}(x, 0)=\mathbf{f}(x)$ and the boundary conditions are

$$
u(0, t)=g_{1}(t), \quad u(1, t)=g_{2}(t)
$$

A semidiscrete approximation is

$$
\frac{d \mathbf{w}_{j}}{d t}=\frac{B}{2 \Delta x}\left(\mathbf{w}_{j+1}-\mathbf{w}_{j-1}\right)
$$

The boundary conditions (3.50) are assumed to be homogeneous:

$$
u_{0}=0, \quad u_{J}=0
$$

The NBSs are

$$
\frac{d v_{0}}{d t}=\frac{u_{1}-u_{0}}{\Delta x}, \quad \frac{d v_{J}}{d t}=\frac{u_{J}-u_{J-1}}{\Delta x} .
$$

Gustafsson [3] used this semidiscrete approximation as an example of a problem that is Lax-Richtmyer stable for homogeneous boundary data but unstable for highly oscillatory non-zero boundary data. We demonstrate that the approximation is GKS unstable because of the presence of a a stationary mode. 
One can show that the NBSs $(3.53 a, b)$ are equivalent to the extrapolation formulas

$$
h(E) u_{-1}=0, \quad p(E) u_{J+1}=0
$$

where

$$
h(E)=1+E^{2}, \quad p(E)=1+E^{-2} .
$$

In deriving (3.54) we have used (3.52). The polynomials $h(\kappa)$ and $p(\kappa)$ are

$$
h(\kappa)=1+\kappa^{2}, \quad p(\kappa)=1+1 / \kappa^{2}
$$

and, therefore,

$$
h(-1 / \kappa)=h(\kappa) / \kappa^{2}, \quad p(-1 / \kappa)=p(\kappa) \kappa^{2} .
$$

Futhermore,

$$
h(-\kappa)=h(\kappa), \quad p(\kappa)=h(1 / \kappa) .
$$

As a consequence of (3.57) and (3.58) there is a stationary mode for the finite-domain problem.

There is a close connection between this example and previous example. In fact, the example of this section can be written as two uncoupled semidiscrete problems of the form given by example 3. The NBS (3.41) is inconsistent but the analogous NBSs (3.54) only appear to be inconsistent, i.e., they are actually consistent.

As in the example of the previous section the eigensolutions of the finite-domain problem can be determined analytically. We look for a solution of (3.51) of the form

$$
\mathbf{w}_{j}=e^{s t} \hat{\mathbf{w}}_{j}
$$

The components $\hat{w}_{j}$ of the eigenvector $\hat{w}$ for the finite-domain problem are given by

$$
\hat{\mathbf{w}}_{j}=a \kappa^{j} \phi_{+}+b(-1 / \kappa)^{j} \phi_{+}+c(-\kappa)^{j} \phi_{-}+d(1 / \kappa)^{j} \phi_{-}
$$

where

$$
\phi_{+}=\left[\begin{array}{l}
1 \\
1
\end{array}\right], \quad \phi_{-}=\left[\begin{array}{r}
1 \\
-1
\end{array}\right] .
$$

The constants $a, b, c, d$ are determined by inserting (3.60) into the boundary conditions (3.52) and (3.54). One obtains a homogeneous linear system and a nontrivial solution exists if and only if

$$
h(\kappa)=0, \quad \kappa^{2 J}=1 .
$$

The polynomial $p(\kappa)$ does not appear here because of identity (3.58b).

We first consider $h(\kappa)=0$ where $h(\kappa)$ is defined by (3.56a). The roots are $\kappa= \pm i$ and for $J$ even these roots also satisfy $(3.62 b)$. For $J$ odd one can show that the roots $\kappa= \pm i$ lead to trivial functions. From (3.62b) $\kappa$ is a root of unity and hence

$$
\kappa=e^{i \pi \ell / J}, \quad \ell=1,2, \cdots, 2 J .
$$


The associated eigenvalues are

$$
\hat{s}_{\ell}=i \sin \left(\frac{\ell \pi}{J}\right), \quad \ell=1,2, \cdots, 2 J
$$

where $\hat{s}=s \Delta x$. For $J$ odd, the eigenvalues (3.64) are identical to the eigenvalues of the circulant matrix associated with the IVP. Furthermore there are no eigenvalues $\hat{s}= \pm i$. But for $J$ even there are are two distinct eigenvalues $\hat{s}= \pm i$.

One can prove by the energy method that the semidiscrete approximation of this section is Lax-Richtmyer stable. But the approximation is GKS unstable because there is a stationary mode. The GKS perturbation analysis is identical to the analysis at the end of example 3 , i.e., an $\epsilon$ perturbation in $\kappa$ yields an $\epsilon^{2}$ perturbation in $\Re(\hat{s})$.

\section{CONCLUSIONS}

Stationary modes for semidiscrete approximations are easy to detect because $q(\kappa)$ and $q(-1 / \kappa)$ have a common factor. For simple approximations the $\kappa$ roots in class (III) defined by (2.1) can be determined analytically and consequently so can the normal modes for the finite-domain problem. This includes, of course, stationary modes for nondissipative approximations where $|\kappa|=1$ independent of $J$. Roots in class $(I)$ and (II) defined by (1.13) cannot be determined analytically and the corresponding normal mode analysis is analytically intractable on the finite domain.

If there is a stationary mode for a nondissipative approximation on a finite-domain problem, then the GKS perturbation test will always indicate the presence of GKS generalized eigenvalue. If in the GKS perturbation test the perturbation in $\kappa$ is the same order as the perturbation in $\Re(\hat{s})$, then the approximation is Lax-Richtmyer unstable in the $L_{2}$ norm. On the other hand, if the the perturbation of $\kappa$ results in a higher order perturbation in $\Re(\hat{s})$, then there is a trivial stationary mode and the approximation is Lax-Richtmyer stable.

If a semidiscrete approximation with a stationary mode is Lax-Richtmyer unstable, then the solution exhibits algebraic growth as the mesh is refined. This is a weak type of instability and the approximation can be thought of as only marginally unstable since the approximation converges in the $L_{2}$ norm for smooth initial and boundary data on a finite domain.

\section{REFERENCES}

[1] B. Gustafsson, H.-O. Kreiss, A. Sundström: "Stability theory of difference approximations for mixed initial boundary value problems. II," Mathematics of Computation, 26, 649-686 (1972).

[2] B. Gustafsson: private communication from preliminary manuscript by B. Gustafsson, H.-O. Kreiss, and J. Oliger (1987).

(3) B. Gustafsson: "Stability analysis for initial-boundary value problems," Lecture Notes in Math., 1005, 128-141, Springer-Verlag, Berlin, (1982).

[4] J.C. Strikwerda: “Initial boundary value problems for the method of lines," J. Comput. Physics, 34, 94-107 (1980). 
[5] L. N. Trefethen: "Instability of difference models for hyperbolic initial boundary value problems," Comm. Pure Appl. Math. 37, 329-367 (1984).

[6] R. F. Warming and R. M. Beam: "Stability of semi-discrete approximations for hyperbolic initial-boundary-value problems: asymptotic estimates," Proceedings of the International Symposium on Computational Fluid Dynamics, Sydney, Australia, North-Holland, Amsterdam (1987). 




